\title{
Patients Prone to Recurrence after Endovascular Treatment: Periprocedural Results of the PRET Randomized Trial on Large and Recurrent Aneurysms
}

J. Raymond, R. Klink, M. Chagnon, S.L. Barnwell, A.J. Evans, J. Mocco, B.L. Hoh, A.S. Turk, R.D. Turner, H. Desal, D. Fiorella, S. Bracard,

A. Weill, F. Guilbert, and D. Roy, on behalf of the PRET Collaborative Group

$0-1$ EBM

\begin{abstract}
BACKGROUND AND PURPOSE: Some patients with large or recurrent aneurysms may be at increased risk of recurrence postcoiling. The Patients Prone to Recurrence after Endovascular Treatment (PRET) trial was designed to assess whether hydrogel coils were superior to platinum coils in these high-risk patients. This article reports periprocedural safety and operator-assessed angiographic results from the PRET trial.
\end{abstract}

MATERIALS AND METHODS: PRET was a pragmatic, multicenter, randomized controlled trial. Patients had $\geq 10$-mm aneurysms (PRET-1) or a major recurrence after coiling of an aneurysm of any size (PRET-2). Patients were randomly allocated to hydrogel or control arms (any platinum coil) by using concealed allocation with minimization. Assist devices could be used as clinically required. Aneurysms could be unruptured or recently ruptured. Analyses were on an intent-to-treat basis.

RESULTS: Four hundred forty-seven patients were recruited (250 PRET-1; 197 PRET-2). Aneurysms were recently ruptured in $29 \%$ of PRET-1 and $4 \%$ of PRET-2 patients. Aneurysms were $\geq 10 \mathrm{~mm}$ in all PRET- 1 and in $50 \%$ of PRET-2 patients. They were wide-neck ( $\geq 4 \mathrm{~mm}$ ) in $70 \%$ and in the posterior circulation in $24 \%$ of patients. Stents were used in $28 \%$ of patients ( $35 \%$ in PRET-2). Coiling was successful in $98 \%$. Adverse events occurred in 28 patients with hydrogel and 23 with platinum coils. Mortality ( $n=2$, unrelated to treatment) and morbidity (defined as $\mathrm{mRS}>2$ at 1 month) occurred in 25 patients ( $5.6 \%$; 12 hydrogel, 13 platinum), related to treatment in 10 (4 hydrogel; 6 platinum) (or $2.3 \%$ of 444 treated patients). No difference was seen between hydrogel and platinum for any of the indices used to assess safety up to at least 30 days after treatment. At 1 month, $95 \%$ of patients were home with a good outcome (mRS $\leq 2$ or unchanged). Operator-assessed angiographic outcomes were satisfactory (complete occlusion or residual neck) in 339 of 447 or $76.4 \%$ of patients, with no significant difference between groups.

CONCLUSIONS: Endovascular treatment of large and recurrent aneurysms can be performed safely with platinum or hydrogel coils.

ABBREVIATIONS: $C C T=$ Cerecyte Coil Trial; DSMC = Data Safety and Monitoring Committee; HELPS = HydroCoil Endovascular Aneurysm Occlusion and Packing Study; MAPS = Matrix and Platinum Science; PI = Principal Investigator; PRET = Patients Prone to Recurrence after Endovascular Treatment

E ndovascular treatment with platinum coils is safe and effective in the treatment of ruptured intracranial aneurysms. Coiling has been shown to improve the 1-year clinical outcome compared

\section{Received May 12, 2014; accepted after revision June 2.}

From the Department of Radiology (J.R., A.W., F.G., D.R.), Centre Hospitalier de I'Université de Montréal and Laboratory of Interventional Neuroradiology (J.R., R.K.), Centre de recherche du Centre Hospitalier de l'Université de Montréal, Notre-Dame Hospital, Montreal, Quebec, Canada; Département de mathématiques et de statistique (M.C.), Université de Montréal, Montreal, Quebec, Canada; Department of Neurological Surgery (S.L.B.), Oregon Health \& Science University, Portland, Oregon; Department of Radiology and Medical Imaging (A.J.E.), University of Virginia Health System, Charlottesville, Virginia; Department of Neurosurgery (J.M., B.L.H.), University of Florida, Gainesville, Florida; Departments of Radiology and Neurosurgery (A.S.T., R.D.T.), Medical University of South Carolina, Charleston, South Carolina; Service de Neuroradiologie Diagnostique et Interventionnelle (H.D.), Centre Hospitalier Universitaire de Nantes, Nantes, France; Department of Neurological Surgery (D.F.), Stony Brook University Medical Center, Stony Brook, New York; and Département de Neuroradiologie Diagnostique et Interventionnelle (S.B.), Centre Hospitalier Universitaire de Nancy, Nancy, France. with surgical clipping. ${ }^{1,2}$ Unfortunately, angiographic recurrences may occur in $10 \%-20 \%$ of patients, necessitating further treatment or generating concern for future rupture or retreatment-related morbidity. ${ }^{3}$ The clinical significance of angiographic recurrence is difficult to determine. A multicenter registry has reported up to $15 \%$ retreatment rates 2 years after coiling of ruptured aneurysms but a yearly rerupture rate of only $0.20 \%$ after the first year. ${ }^{4}$ Similar findings were reported after the Inter-

Paper previously presented in part at: Annual Meeting of the American Society of Neuroradiology and the Foundation of the ASNR Symposium, May 17-22, 2014; Montreal, Quebec, Canada.

Please address correspondence to Jean Raymond, MD, CHUM, Notre-Dame Hospital, Department of Radiology, 1560 Sherbrooke East, Pavilion Simard, Suite Z12909, Montreal, Quebec, Canada H2L 4M1; e-mail: jean.raymond@umontreal.ca

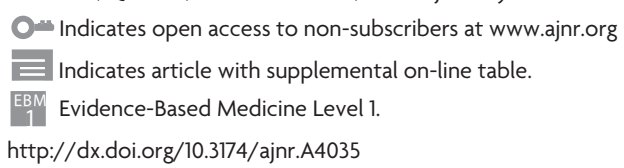

AJNR Am J Neuroradiol 35:1667-76 Sep 2014 www.ajnr.org 
national Subarachnoid Aneurysm Trial. ${ }^{5}$ If posttreatment ruptures have been rare, we must remember that such low rates were observed in patients who were followed and retreated when recurrences occurred. ${ }^{6}$ The management of unruptured aneurysms remains controversial, but coiling is increasingly used, even though it has never been proved superior to surgery ${ }^{7}$ or to observation. ${ }^{8}$ Because the efficacy of coiling in preventing aneurysmal ruptures in patients with unruptured aneurysms has never been shown, angiographic occlusion of aneurysms remains the most frequent surrogate marker of clinical efficacy. ${ }^{9}$ Recurrence after endovascular treatment may affect patients with ruptured or unruptured aneurysms.

If the endovascular approach is to be improved in terms of long-term efficacy, this improvement should preferably be accomplished without compromise regarding procedural safety. Second generations of coils have been introduced for this purpose. ${ }^{10}$ However, there is no rigorous evidence that coated coils improve the angiographic or clinical outcomes. Two randomized trials have failed to demonstrate a benefit from the use of coils coated with or containing resorbable suture material. ${ }^{11,12}$ Hydrogel coils were initially designed to improve volumetric filling of the aneurysm with an expansive material that should fill a higher percentage of the aneurysm lumen than standard platinum coils, aiming to improve aneurysm stability after treatment. One trial comparing hydrogel and platinum coiling in 500 patients with aneurysms showed a lower proportion of core laboratory-adjudicated angiographic recurrences in the hydrogel arm at follow-up (a secondary outcome measure) but no significant difference in the composite primary outcome measure. ${ }^{13}$ The Patients Prone to Recurrence after Endovascular Treatment (PRET) trial was designed before the aforementioned trials had completed recruitment and follow-up of patients and before results were published. PRET was designed with the premise that some patients were at such a high risk of aneurysm recurrence at follow-up (in the range of $50 \%$ ) that this risk should be revealed to patients before treatment and perhaps a different approach should at least be offered. Furthermore, given the unknown risks of alternative coils and the lack of evidence that they are beneficial, treatment with these coils should be offered only within the context of a randomized trial, until convincingly shown to be superior. ${ }^{14} \mathrm{~Pa}-$ tients identified to be at high risk for recurrences were those with a large aneurysm ( $\geq 10 \mathrm{~mm}$ or PRET-1 patients) and those already presenting with a recurrence after previous coiling (PRET2). ${ }^{11,13,15}$ We aimed to establish whether the use of hydrogel coils for high-risk patients improved angiographic outcomes compared with bare platinum coils, without increasing procedural risks. The present report focuses on operator-assessed immediate treatment success and procedural morbidity and mortality up to 1 month after the procedure. The primary outcome of the trial will be reported once the 18-month follow-up is complete.

\section{MATERIALS AND METHODS}

PRET is an investigator-led, pragmatic, multicenter, international, randomized controlled trial comparing a policy of using hydrogel versus bare platinum coils in the endovascular treatment of intracranial aneurysms in patients prone to recurrence. There were 25 participating centers from 6 countries (United States,
Canada, United Kingdom, France, Chile, Japan). The ClinicalTrials. gov registration number from the US National Institutes of Health is NCT00626912. All trial sites had local institutional review board approval. All patients (or legal representatives) signed a standardized informed consent form.

\section{Patients}

Patients with an intracranial aneurysm requiring endovascular treatment by the neurovascular team, but prone to recurrence, were eligible for the trial; the aneurysm could be ruptured (World Federation of Neurological Societies $\leq 3$ ) or unruptured. Such patients fell into 1 of 2 groups: PRET-1, with a large aneurysm (longest dimension, $\geq 10 \mathrm{~mm}$, including any thrombosed portion), never treated; PRET-2, with an aneurysm of any size, presenting with a major recurrence after previous coiling. ${ }^{15}$ A recurrence qualified as "major" if it was "saccular and its size would theoretically permit re-treatment with coils." ${ }^{15}$ There were few selection criteria: the patient was 18 years of age or older; life expectancy was $>2$ years; anatomy was such that endovascular treatment was considered possible with both types of coils; the endovascular operator was satisfied with using either type of coil, but no other type; and the patient or authorized representative had given fully informed consent and had signed the consent form. Patients were not eligible if they met any of the following criteria: the presence of other aneurysms requiring treatment during the same session; the presence of an associated cerebral arteriovenous malformation; the primary intent of the procedure being parent vessel occlusion without simultaneous endovascular coiling of the aneurysm; and any absolute contraindication to endovascular treatment, angiography, or anesthesia.

\section{Randomization}

Randomized allocation was through the Web-based PRET application package (designed by MediSciNet, Stockholm, Sweden), ensuring that allocation was concealed before the decision to include a patient. From the moment of randomization, the patient was in the trial and accounted for in the analysis (intention-totreat). PRET-1 and PRET-2 patient groups were randomized separately; treatment groups were matched according to the following minimization criteria: rupture status (yes, no); if the aneurysm was unruptured, planned use of an adjunct device (yes, no).

\section{Embolization Procedure}

Endovascular operators were not blinded to treatment allocation. Patients were masked to allocation unless they specifically requested otherwise. Standard local procedures were followed. Any locally approved bare platinum coil with controlled detachment was permitted, as were any assist devices believed necessary by the operator, provided they had local regulatory approval, excluding flow diverters, irrespective of intended use indicated at randomization. Antiplatelet and anticoagulation regimens were left to individual operator judgment, according to the clinical practice at each site.

When treatment allocation was to "platinum," types of coils other than bare platinum were forbidden. When treatment allocation was to "hydrogel," any coil of the hydrogel family was al- 
lowed but any bare platinum coil could also be used if the operator believed it was in the patient's best interest. Recommendations concerning hydrogel coil use pertaining to type, size, and sequence of introduction were issued but not enforced. No minimum percentage of hydrogel coils was prescribed; the protocol required "the substitution, as far as possible, of platinum by hydrogel coils, the operator always being allowed to use the coils he/she believes is appropriate at any time during the procedure." ${ }^{14}$ Other technical considerations such as steaming of hydrogel coils and the type of bare platinum coil were left entirely to the operator's discretion. The goal of the procedure was to occlude the aneurysm as completely as possible, keeping the risks of the procedure as low as possible. ${ }^{14}$

\section{Trial Monitoring}

Monitoring of trial data quality was Web-based and was performed by periodic review of data stored in the data base. Blinded data were prepared for periodic reviews at prespecified intervals by an independent Data Safety and Monitoring Committee (DSMC) to ensure patient safety. A DSMC Charter predefined all trial-monitoring procedures. Unblinding criteria were prespecified in the DSMC Charter, but the need for unblinding did not arise during the conduct of the trial.

\section{Data Collection}

Data capture and management were held independent of the Steering Committee, sponsor, and funder on the secure servers of MedSciNet, ensuring FDA 21 Code of Federal Regulations Part 11, Good Clinical Practice requirements compliance. The Registration form included the following: demographics (age, sex); other aneurysms; subarachnoid hemorrhage; date and World Federation of Neurological Societies grade at the time of randomization if SAH occurred; mRS grade (if no SAH); whether the target aneurysm was a symptomatic, additional or incidental aneurysm; aneurysm location and dimensions (maximum size, length, width, and neck size); and planned use of adjunct devices. The procedure form included the following: the use of adjunct devices (mainly stents; balloon-assistance, virtually in routine use for such difficult aneurysms in many PRET centers; these were not recorded); aneurysm occlusion grade based on the Montreal grading system ${ }^{16}$ as judged by the operator; medication used during the procedure; and clinical outcome and complications during treatment (categorized as hemorrhagic, thromboembolic, or other). The total length of each type of coil was also recorded. Because the HydroSoft, HydroFrame, and HydroFill (MicroVention, Tustin, California) were marketed at different times in July 2008, April 2011, and April 2012, respectively, after the launch of the PRET trial in June 2007, a single new entry mentioning "hydrogel-core" coils was added to the Case Report Forms in December 2007, to indicate the use of those newer coil types.

The discharge form included dates of admission and discharge, discharge destination (home, hospital, rehabilitation center), whether the patient was discharged with a prescription for antiplatelet therapy, any imaging performed after the procedure, any new neurologic or imaging changes, whether ventricular shunting was performed during hospitalization, any adverse event, and mRS score at discharge. Follow-up forms (at 1, 6, 12, and 18 months) were all designed on the same pattern, including questions regarding new symptoms (including headaches, fever, or chills), neurologic events, new imaging findings, other treatments, any admission since the last assessment, and the modified Rankin Scale as the clinical outcome measure. Adverse events were reported at any time during the trial, and automatic notification was sent to the study monitor immediately. The present article is limited to procedural results, including events occurring within 30 days of treatment (or more if within the initial admission) as reported in the procedure and discharge forms $(n=444$, including 14 patients with no further follow-up), 1-month follow-up (392 patients) form, or later (38 patients). Imaging studies (procedural and follow-ups) were anonymized and sent to the core laboratory (P.W. White, Newcastle University, Newcastle upon Tyne, United Kingdom) for central adjudication, and follow-up angiographic results will be the object of a future publication. The angiographic results given here were the ones reported by local investigators at the end of treatment.

\section{Safety End Points}

The protocol hypothesized that "the number of adverse events was similar for both hydrogel and platinum groups, and that morbidity and mortality related to treatment remained unchanged for both PRET-1 and PRET-2 patients." ${ }^{14}$ All adverse events were reviewed by an independent Adverse Event Committee and categorized as the following: 1) related to the illness (SAH for example), 2) related to coil embolization, or 3) unrelated. The protocol also prespecified that morbidity would be defined per patient, according to the mRS score. Adverse events reports and individual case report forms were cross-checked to determine the safety of coil embolization (periprocedure and $\geq 30$ days after if events occurred during the same admission) for each patient, categorized as the following: 1) death or dependency (mRS >2) at 30 days (unrelated to the coiling procedure when no adverse events were reported; related if any serious adverse event was reported), 2 ) any stroke or neurologic event periprocedure or within 30 days, without dependency (mRS 0-2), 3) any procedural or predischarge complication or adverse event, and 4) uneventful hospitalization and procedure (no complication). Other safety indices are also reported, including procedural complications (sorted as hemorrhagic, thromboembolic, or others), neurologic deteriorations after the procedure or at discharge, $\mathrm{mRS}$ at discharge and 1 month, discharge destination, location at 1 month, and length of hospitalization (mean-median number of days and number of patients hospitalized for $>5$ days for unruptured aneurysms and $>15$ days for $\mathrm{SAH}$ ). The number and severity of adverse events are also reported per group. To detect inflammatory complications potentially related to coils, we reviewed all adverse events within 30 days: new imaging findings (when performed and reported); new headaches; fever or chills or cranial nerve deficits up to 30 days after the procedure; and the number of patients in whom ventricular drainage was performed.

\section{Statistical Methods}

All analyses were performed by the trial statistician (M.C.), according to the published trial protocol. ${ }^{14}$ Analyses were intent-to- 


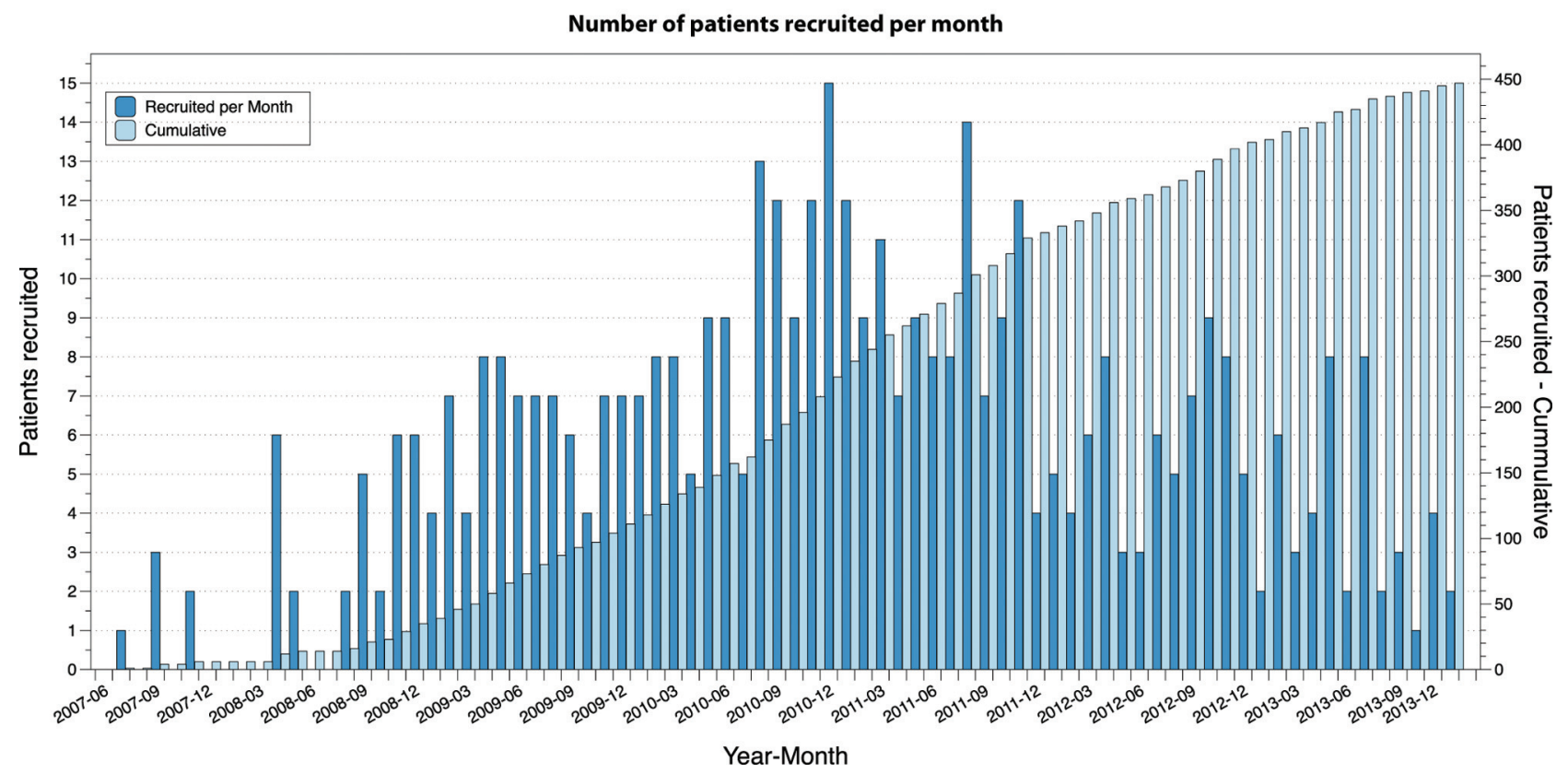

FIG 1. Recruitment. Notice how recruitment decreased progressively from December 2010 to December 2013, a possible sign of case selection.

treat. Categoric variables were compared by using the Fisher exact test, and continuous variables, by using the Student $t$ test. To evaluate the possibility of different results for PRET-1 and PRET-2, we stratified descriptive and safety analyses by group. All analyses were done with SPSS, Version 21 (IBM, Armonk, New York) by using a significance level of $5 \%$.

\section{Roles of the Sponsor and Funding Source}

The trial was sponsored by the Centre Hospitalier de l'Université de Montréal and funded by MicroVention Terumo Incorporated. The sponsor and funder had no part in study design, data collection, analysis, or reporting and had no direct or indirect access to the data or source documents. The Steering Committee bears the sole responsibility for all aspects of the trial.

\section{RESULTS}

\section{Recruitment}

Recruitment started in June 2007, but only 6 patients were included in a single center by the end of the year. Recruitment increased to reach a peak rate of 15 patients per month in December 2010, and slowly decreased thereafter (Fig 1). On December 13, 2013, when close to 250 patients had been recruited in PRET-1, the Steering Committee decided to stop recruitment before reaching the target number of patients for PRET-2 ( $n=197$ instead of 250) because of the following: 1) recruitment had decreased, particularly for patients in PRET-2 during the previous years; 2) the trial was already 2 years behind schedule; and 3) provisions had to be made to continue monitoring and cover compensations to participating sites for the 18-month follow-up data. This decision was made despite the recommendation of the DSMC meeting of February 2013 to continue recruitment. The registration Web site was closed to patient entry and randomization on January 15,2014 . By that date, target recruitment had been reached for PRET-1 $(n=250)$. The part of the data base containing baseline and early safety information was locked, but we are still collecting
6-, 12-, and 18-month follow-up data. A total of 447 patients were randomized by 25 centers in 6 countries ( 250 in PRET- 1 and 197 in PRET-2).

\section{Baseline Characteristics}

Baseline characteristics of patients and aneurysms recruited in both PRET-1 and -2 are shown in On-line Table 1. There was no significant difference between the hydrogel and platinum groups.

\section{Flow Chart}

All patients are included in the present analyses. Results up to 30 days (or events during the initial admission if longer) were collected and reported in the procedural, discharge, and 1-month follow-up forms for all 444 treated patients, as depicted in Fig 2.

\section{Withdrawal, Failures, and Protocol Deviations}

Three patients (2 PRET-1 and 1 PRET-2) were withdrawn before any treatment was attempted (1 protocol violation [World Federation of Neurological Societies 4 after SAH]), 1 PRET-1 aneurysm judged untreatable, 1 patient in PRET-2 in whom no true recurrence was found; all 3 allocated to hydrogel). Treatment was attempted, but coils were not deployed in 4 patients in PRET-1 (1 hydrogel; 3 platinum) and 4 in PRET-2 (4 hydrogel) (or 1.8\% of patients). Failure to catheterize branches for balloon-assisted or stent-assisted coiling or unstable first coils was the cause of these failures. All 8 patients were discharged home without complication within 2 days ( 6 with mRS 0 ) or 20 days ( $1 \mathrm{mRS} 1$ and $1 \mathrm{mRS}$ 0 , the last 2 patients after $\mathrm{SAH}$ ).

Three patients in PRET-1 were not treated as allocated: One patient allocated to hydrogel was treated with only platinum coils $(87 \mathrm{~cm})$. One patient allocated to platinum coils was treated with some hydrogel coils $(118 \mathrm{~cm}$ of platinum; $67 \mathrm{~cm}$ of hydrogel coils). Cerecyte coils ( $247 \mathrm{~cm}$; Codman Neurovascular, Raynham, 


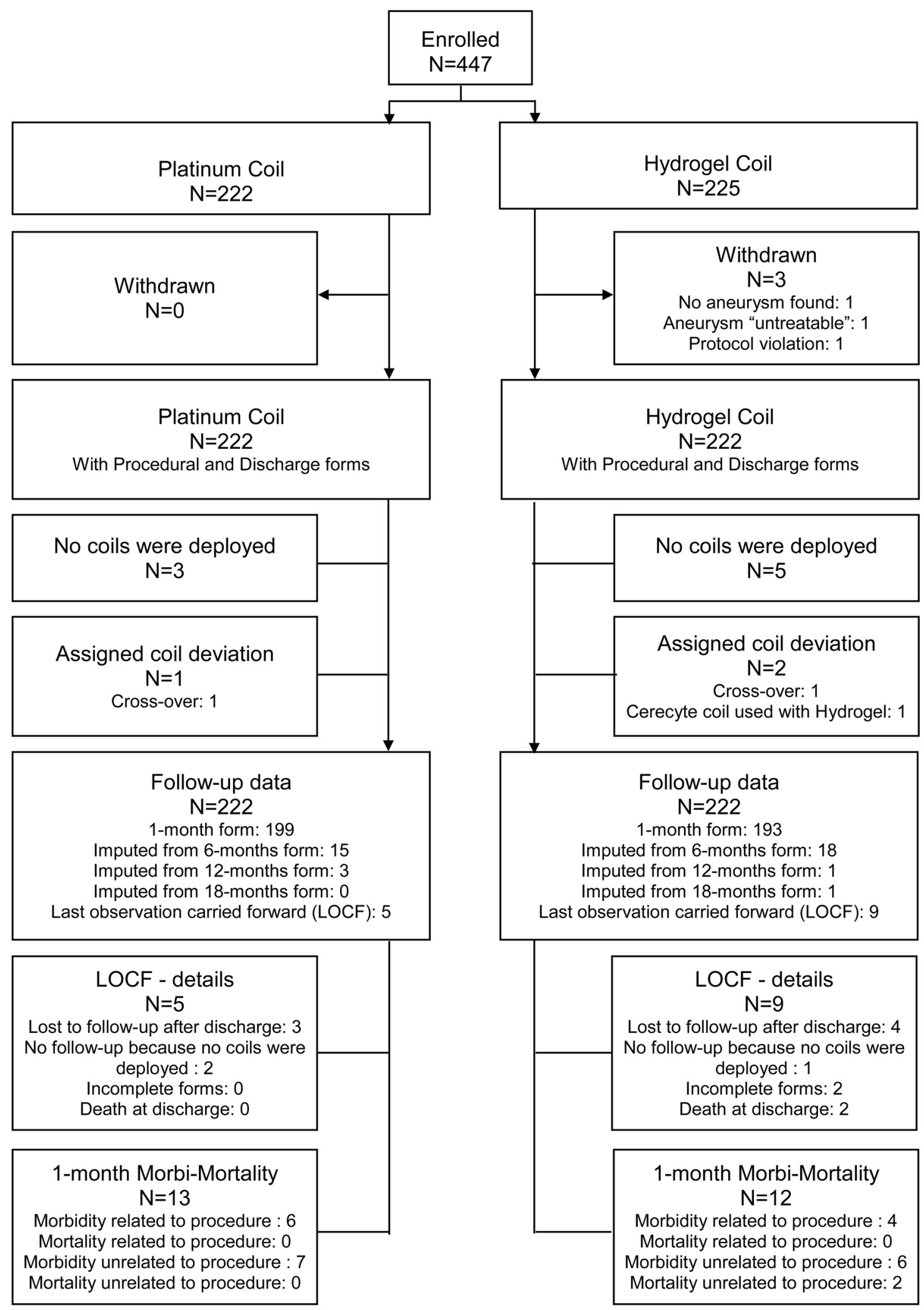

FIG 2. Flow chart. Diagram shows patient flow from randomization to periprocedural safety end points.

Massachusetts) were used in 1 other patient allocated to and treated with hydrogel coils (237-cm hydrogel; 50-cm platinum). None of these 3 patients had any complications.

The mean total length of coils was $129 \mathrm{~cm}$ for platinum cases
(178 cm for PRET-1; $68 \mathrm{~cm}$ for PRET-2) and $149 \mathrm{~cm}$ for hydrogel cases ( $125 \mathrm{~cm}$ for hydrogel; $24 \mathrm{~cm}$ for platinum) (158-cm hydrogel $+33-\mathrm{cm}$ platinum for patients in PRET-1; 83-cm hydrogel +12 -cm platinum for those in PRET-2). In 202 of 222 patients 


\begin{tabular}{|c|c|c|c|c|c|c|}
\hline & \multicolumn{2}{|c|}{ PRET-1 } & \multicolumn{2}{|c|}{ PRET-2 } & \multicolumn{2}{|c|}{ PRET } \\
\hline & Platinum & Hydrogel & Platinum & Hydrogel & Platinum & Hydrogel \\
\hline \multicolumn{7}{|l|}{ Death } \\
\hline Total & 0 & $2(1.6 \%)$ & 0 & 0 & 0 & $2(0.9 \%)$ \\
\hline Treatment-related & 0 & 0 & 0 & 0 & 0 & 0 \\
\hline \multicolumn{7}{|l|}{ Morbidity (mRS > 2) } \\
\hline Total & $11(8.8 \%)$ & $5(4.0 \%)$ & $2(2.1 \%)$ & $5(5.0 \%)$ & $13(5.9 \%)$ & $10(4.4 \%)$ \\
\hline Treatment-related & $5(4.0 \%)$ & $2(1.6 \%)$ & $1(1.0 \%)$ & $2(2.0 \%)$ & $6(2.7 \%)$ & $4(1.8 \%)$ \\
\hline Any stroke & $11(8.8 \%)$ & $5(4.0 \%)$ & $8(8.2 \%)$ & $9(9.0 \%)$ & $19(8.6 \%)$ & $14(6.2 \%)$ \\
\hline Any complication & $8(6.4 \%)$ & $4(3.2 \%)$ & $1(1.0 \%)$ & $2(2.0 \%)$ & $9(4.1 \%)$ & $6(2.7 \%)$ \\
\hline No complication & $95(76.0 \%)$ & $107(85.6 \%)$ & $86(88.7 \%)$ & $83(83.0 \%)$ & $181(81.5 \%)$ & $190(84.4 \%)$ \\
\hline Withdrawn & 0 & $2(1.6 \%)$ & 0 & $1(1.0 \%)$ & 0 & $3(1.3 \%)$ \\
\hline Fisher exact test $P$ value & \multicolumn{2}{|c|}{.052} & \multicolumn{2}{|c|}{.612} & \multicolumn{2}{|c|}{.174} \\
\hline
\end{tabular}

allocated to hydrogel (91\%), more than two-thirds of the total coil length was hydrogel.

\section{Periprocedural Outcomes}

Procedural and discharge case report forms were available for all 444 treated patients, and follow-up at $\geq 1$ month, in 430 (97\% of treated patients).

There is no follow-up observation beyond discharge for 2 patients due to death and 12 other patients for whom no complication or adverse event was reported. These patients are included in the 1-month analyses (last observation carried forward). Three previously mentioned failures ( 1 hydrogel, 2 platinum) had no further follow-up. Nine other patients (6 hydrogel; 3 platinum) were discharged home after successful procedures, after 1 day ( $n=6$; mRS 0 for 5 patients and mRS 1 [hydrogel] for 1 patient) or 7 days $(n=1$; mRS 0 [platinum]) for 7 patients with unruptured aneurysms and after 11 days (hydrogel; mRS 0 ) or 13 days (platinum; mRS 2) for 2 patients with SAH.

The 1-month case report forms were available in 392 patients. In 38 other patients, the follow-up information was taken from the 6-month $(n=33$; hydrogel $=18$; platinum $=15)$, 12-month $(n=4$; hydrogel $=1$; platinum $=3)$, or 18 -month $(n=1$; hydrogel) case report forms (Fig 2).

Periprocedural outcomes are summarized in Table 1. Two patients in PRET-1 (0.45\%) died in the hospital 13 and 56 days after SAH, 11 and 55 days after uneventful, completed procedures (both hydrogel), one from vasospasm (related to the initial SAH), the other from cardiac arrest while on dialysis (unrelated).

Five patients were dependent before admission (1 hydrogel; 4 platinum). Twenty-three patients (5.2\%) became and were still dependent 1 month after admission (16 PRET-1; 7 PRET-2; 10 hydrogel; 13 platinum). Of those, 13 patients ( 5 hydrogel; 8 platinum) were dependent at 1 month from SAH or aneurysm-related events ( 4 from vasospasm [ 1 hydrogel; 3 platinum]; 1 from hydrocephalus [platinum]; or without adverse events being reported [ 8 patients; 4 hydrogel; 4 platinum]).Ten patients were dependent from treatment-related complications, such as thromboembolic events ( $n=5 ; 1$ hydrogel; 4 platinum), coil perforation ( $n=2 ; 2$ hydrogel; 0 platinum), or postoperative strokes reported at discharge or 1 month ( $n=3 ; 1$ hydrogel; 2 platinum).

Strokes revealed by neurologic deficits without dependency or by asymptomatic imaging findings were found in 33 (7.4\%) additional patients (14 hydrogel; 19 platinum).
Procedural complications were reported in 15 additional patients (6 hydrogel; 9 platinum), but without clinical or imaging consequences.

Technical complications related to coils were reported in 9 patients ( 6 hydrogel; 3 platinum) including coil stretching, fracturing, or premature detachment. Four of these events necessitated coil retrieval with a snare ( 3 hydrogel; 1 platinum). In 1 patient (hydrogel), the technical complication was associated with a clinical deterioration (mRS 3 at 1 month).

Other indices of safety, such as procedural complications, neurologic deteriorations, length of hospitalization, mRS, and location at discharge and 1 month, are summarized in Table 2. There was no significant difference between hydrogel and platinum for any of these comparisons.

\section{Other Adverse Events}

Adverse events within 30 days, reported in 51 patients ( 28 hydrogel; 23 platinum), were serious in 27 cases ( 14 hydrogel; 13 platinum). In addition to previously reported procedural events, 3 patients presented with transient ischemic symptoms in a delayed fashion (1 hydrogel; 2 platinum) and 2 patients (both hydrogel) had femoral artery complications, 1 serious, with retroperitoneal hematoma. Two hemorrhagic complications possibly related to antiplatelet therapy (epistaxis, gastrointestinal hemorrhage) occurred in a delayed fashion (1 hydrogel; 1 platinum).

Seven patients had new or increasing cranial nerve deficits immediately after the procedure ( 3 patients with cavernous $[n=$ 2] or ophthalmic aneurysms) or 6-18 days later (2 patients with hydrogel with ophthalmic or carotid bifurcation aneurysms; 2 patients with platinum with ophthalmic or midbasilar aneurysms). None were serious events; they were variously labeled as "mass effect" or "inflammation" and treated with steroids.

Increased headaches were reported at 1 month in 63 patients (31 hydrogel; 32 platinum); and fever or chills, in 6 patients (4 hydrogel; 2 platinum) without neurologic events or imaging findings. Ventricular drainage was reported in 9 patients ( 5 hydrogel; 4 platinum $)$ at discharge, all after the initial SAH $(n=8)$ or after coil perforation during the procedure $(n=1)$.

\section{Angiographic Results and Exploratory Analyses}

Immediate angiographic results assessed by local investigators are presented for 447 enrolled patients. They are summarized in Table 3. There was no significant difference between groups $(P=.28)$. 
Table 2: Safety indices reported at time of the procedure and during follow-up

\begin{tabular}{|c|c|c|c|c|c|c|}
\hline & \multicolumn{2}{|c|}{ PRET-1 } & \multicolumn{2}{|c|}{ PRET-2 } & \multicolumn{2}{|c|}{ PRET } \\
\hline & Platinum & Hydrogel & Platinum & Hydrogel & Platinum & Hydrogel \\
\hline \multicolumn{7}{|l|}{ Procedural complications } \\
\hline Thromboembolic & $10(8.0 \%)$ & $6(4.9 \%)$ & $1(1.0 \%)$ & $3(3.0 \%)$ & $11(5.0 \%)$ & $9(4.1 \%)$ \\
\hline Hemorrhagic & $2(1.6 \%)$ & $1(0.8 \%)$ & $1(1.0 \%)$ & $1(1.0 \%)$ & $3(1.4 \%)$ & $2(0.9 \%)$ \\
\hline Other & $4(3.2 \%)$ & $6(4.9 \%)$ & $4(4.1 \%)$ & $3(3.0 \%)$ & $8(3.6 \%)$ & $9(4.1 \%)$ \\
\hline Anti-GP IIb/IIla used during embolization & $8(6.4 \%)$ & $11(8.9 \%)$ & $7(7.2 \%)$ & $4(4.0 \%)$ & $15(6.8 \%)$ & $15(6.8 \%)$ \\
\hline Clinical deterioration at end of procedure & $5(4.0 \%)$ & $1(0.8 \%)$ & $2(2.1 \%)$ & $3(3.0 \%)$ & $7(3.2 \%)$ & $4(1.9 \%)$ \\
\hline \multicolumn{7}{|l|}{ Hospitalization } \\
\hline Days (median) (min) (max) & $2(1)(94)$ & $1(0)(70)$ & $1(0)(51)$ & $1(0)(30)$ & $1(0)(94)$ & $1(0)(70)$ \\
\hline Days $>5$ for unruptured aneurysms & $9(7.2 \%)$ & $7(5.7 \%)$ & $5(5.2 \%)$ & $5(5.1 \%)$ & $14(6.3 \%)$ & $12(5.4 \%)$ \\
\hline Days $>15$ for ruptured aneurysms & $10(8.0 \%)$ & $10(8.1 \%)$ & $1(1.0 \%)$ & 0 & $11(5.0 \%)$ & $10(4.5 \%)$ \\
\hline New imaging findings at discharge & $17(13.6)$ & $11(8.9 \%)$ & $7(7.2 \%)$ & $9(9.1 \%)$ & $24(10.8 \%)$ & $20(9.0 \%)$ \\
\hline $\mathrm{mRS}>2$ at discharge & $17(13.6 \%)$ & $9(7.3 \%)$ & $1(1.0 \%)$ & $5(5.1 \%)$ & $18(8.1 \%)$ & $14(6.3 \%)$ \\
\hline \multicolumn{7}{|l|}{ Discharge destination } \\
\hline Home & $107(85.6 \%)$ & $108(87.8 \%)$ & $95(97.9 \%)$ & $92(92.9 \%)$ & $202(91.0 \%)$ & $200(90.1 \%)$ \\
\hline Other than home & $18(14.4 \%)$ & $15(12.2 \%)$ & $2(2.1 \%)$ & $7(7.1 \%)$ & $20(9.0 \%)$ & $22(9.2 \%)$ \\
\hline $\mathrm{mRS}>2$ at 1 month & $11(8.8 \%)$ & $6(4.9 \%)$ & $3(3.1 \%)$ & $5(5.1 \%)$ & $14(6.3 \%)$ & $11(5 \%)$ \\
\hline \multicolumn{7}{|l|}{ Location at 1 month } \\
\hline Home & 117 (93.6\%) & $116(94.3 \%)$ & 95 (97.9\%) & $95(96.0 \%)$ & $212(95.5 \%)$ & $211(95.0 \%)$ \\
\hline Other than home & $8(6.4 \%)$ & $7(5.7 \%)$ & $2(2.1 \%)$ & $4(4.0 \%)$ & $10(4.5 \%)$ & $11(5 \%)$ \\
\hline \multicolumn{7}{|l|}{$A E$} \\
\hline No. reported & 16 & 18 & 7 & 10 & 23 & 28 \\
\hline No. (\%) serious & $10(62 \%)$ & $8(44.4 \%)$ & $3(42.9 \%)$ & $6(60 \%)$ & $13(56.5 \%)$ & $14(50 \%)$ \\
\hline \multicolumn{7}{|l|}{ AE attribution (No.) (\% of total No. reported) } \\
\hline Related to treatment & $14(87.5 \%)$ & 14 (77.8\%) & 7 (100\%) & 10 (100\%) & $21(91.3 \%)$ & $24(85.7 \%)$ \\
\hline Related to aneurysm & $2(12.5 \%)$ & $3(16.7 \%)$ & 0 & 0 & $2(8.7 \%)$ & $3(10.7 \%)$ \\
\hline Unrelated & 0 & $1(5.5 \%)$ & 0 & 0 & 0 & $1(3.6 \%)$ \\
\hline
\end{tabular}

Note:-Anti-GP IIb/IIla indicates antiglycoprotein Ilb/Illa; AE, adverse events; min, minimum; max, maximum.

Table 3: Operator-assessed immediate angiographic results

\begin{tabular}{|c|c|c|c|c|c|c|}
\hline & \multicolumn{2}{|c|}{ PRET-1 } & \multicolumn{2}{|c|}{ PRET-2 } & \multicolumn{2}{|c|}{ PRET } \\
\hline & Platinum & Hydrogel & Platinum & Hydrogel & Platinum & Hydrogel \\
\hline No coiling, for any reason & $3(2.4 \%)$ & $3(2.4 \%)$ & 0 & $5(5.0 \%)$ & $3(1.4 \%)$ & $8(3.6 \%)$ \\
\hline Residual aneurysm & $32(25.6 \%)$ & $39(31.2 \%)$ & $12(12.4 \%)$ & $14(14.0 \%)$ & $44(19.8 \%)$ & $53(23.6 \%)$ \\
\hline Residual neck & $48(38.4 \%)$ & $48(38.4 \%)$ & $40(41.2 \%)$ & $40(40.0 \%)$ & $88(39.6 \%)$ & $88(39.1 \%)$ \\
\hline Complete obliteration & $42(33.6 \%)$ & $35(28.0 \%)$ & $45(46.4 \%)$ & $41(41.0 \%)$ & $87(39.2 \%)$ & $76(33.8 \%)$ \\
\hline Fisher exact test $P$ value & \multicolumn{2}{|c|}{.723} & \multicolumn{2}{|c|}{.151} & \multicolumn{2}{|c|}{.280} \\
\hline
\end{tabular}

Exploratory analyses of procedural morbidity and immediate angiographic results, for all unruptured aneurysms, for carotid aneurysms, for patients treated by stent-assisted coiling, and for all patients according to aneurysm size categories, are provided in On-line Tables 2-5. There was no difference between patients receiving hydrogel and platinum.

\section{DISCUSSION}

The main findings of this report are the following: 1) coiling of large and recurrent aneurysms could be performed in 436 or $98 \%$ of 447 patients; treatment-related morbidity and mortality, defined as $\mathrm{mRS}>2$ at 1 month and attributed to treatment, occurred in $2.3 \%(1.2 \%-4.1 \%)$ (10 of 444 treated patients; Table 1 ); 2) there was no significant difference between hydrogel and platinum coiling for any of the safety indices we reviewed (Table 2);3) immediate angiographic results, judged by local investigators, were satisfactory (complete occlusion or residual neck) in 339 of 447 or $76.4 \%$ of patients, with no significant difference between groups (Table 3 ).

The selection of patients recruited in the PRET trial differs (by design and as a consequence of the design) from the patients recruited in the 4 other major randomized trials on aneurysm coil-

ing: None of the other trials included patients presenting with recurrences (an exclusion criterion for most trials); $44 \%$ of patients in PRET were PRET-2. PRET aneurysms were larger (78\% of PRET, or all patients in PRET-1 and 50\% of those in PRET-2 had $\geq 10 \mathrm{~mm}$ aneurysms); the proportion of aneurysms of $\geq 10$ mm was $10 \%$ in the International Subarachnoid Aneurysm Trial, ${ }^{1}$ $12 \%$ in the Cerecyte Coil Trial (CCT), ${ }^{17} 21 \%$ in the Matrix and Platinum Science (MAPS) ${ }^{11}$ trial, and $24 \%$ in the HydroCoil Endovascular Aneurysm Occlusion and Packing Study (HELPS). ${ }^{18}$ Aneurysms were wide-neck ( $\geq 4 \mathrm{~mm}$ ) in $70 \%$ of patients in PRET, compared with $37 \%$ in MAPS, $32 \%$ in HELPS, and $19 \%$ in CCT. Stents were used in $28 \%$ of patients in PRET (35\% of those in PRET-2), 22\% in MAPS, $20 \%$ in HELPS, and $0.6 \%$ of those in the CCT. Posterior circulation aneurysms were more frequent in PRET (108 or $24 \%$, including 73 basilar bifurcation aneurysms, compared with $12 \%$ in CCT, $13 \%$ in MAPS, and only $2.7 \%$ in the International Subarachnoid Aneurysm Trial). The proportion of patients treated for ruptured aneurysms was approximately $36 \%$ in MAPS, $48 \%$ in CCT, $53 \%$ in HELPS, $100 \%$ in the International Subarachnoid Aneurysm Trial, but only 18\% in PRET (29\% in PRET-1; 4\% in PRET-2).

These differences are expected to impact clinical results, in- 
cluding periprocedural complications. For example, procedural aneurysmal ruptures or perforations may be less frequent during the treatment of larger, unruptured aneurysms. ${ }^{17,19,20}$ Conversely, thromboembolic complications may be more frequent, though size as a potential risk factor for complications did not reach a prespecified $P$ value of .01 in a previous meta-analysis of coiling of unruptured aneurysms. ${ }^{9}$ Hemorrhagic complications have occurred in approximate proportion to the number of patients treated acutely (approximately 2\% in MAPS, $4 \%$ in CCT and HELPS, but only $1.1 \%$ in PRET). Thromboembolic complications have varied from $4 \%$ to $28 \%$ of cases in the literature (between 5\% and 10\% in the CCT, MAPS, and HELPS studies), depending on the case selection, definitions, and methods of detection. ${ }^{21}$ Many thromboembolic complications detected at the time of coiling (3\%-4\% in MAPS; $5 \%-7 \%$ in CCT; $5 \%-10 \%$ in HELPS; $4.5 \%$ in PRET) may be successfully managed without clinical consequence, while others may occur, sometimes unnoticed, immediately after treatment or be confounded with vasospasm-related strokes. Fifty-six neurologic events or imaging findings (or $12.6 \%$ of patients) up to 1 month after treatment were consistent with any stroke (symptomatic or not, treatmentrelated or not) in PRET. There was no difference between the hydrogel and platinum groups.

The morbidity associated with coiling is perhaps best estimated when we focus on patients with unruptured aneurysms. The overall 1-month treatment-related mortality and morbidity ( $\mathrm{mRS}>2$ ) was $0 \%$ and $2.3 \%(1.2 \%-4.4 \%)$ for 361 patients in PRET-1 or -2 with unruptured aneurysms and $0 \%$ and $1.7 \%$ $(0.5 \%-4.8 \%)$ for the 178 patients in PRET-1 treated for previously untreated large unruptured aneurysms, including $122 \mathrm{pa}-$ tients treated with stent-assisted coiling (associated with $4.1 \%$ [1.8\%-9.2\%] morbidity [On-line Tables 2 and 4]).

Technical problems during coil deployment (stretching or premature detachment) may have been more frequent with hydrogel $(n=6)$ than platinum $(n=3)$, but we did not find a statistical difference between coils. The $20 \%$ reduction of the total length of coils deployed when hydrogel was used in the HELPS trial $^{13}$ was not confirmed in PRET. This difference may be due, in part, to the availability of smaller hydrogel-core finishing coils.

Inflammatory problems (cranial nerve deficits, meningitislike syndromes, and hydrocephalus in patients with unruptured aneurysms) have previously been reported with the use of hydrogel coils. ${ }^{13,18,22-25}$ Even though reports such as "increased mass effect" or "inflammation" were slightly more frequent with hydrogel (5 versus 2 platinum), we did not find a significant difference with platinum or did not show an impact on treatment morbidity. Differences in immediate angiographic outcomes, with a higher (but not statistically significant) proportion of residual aneurysms after hydrogel coiling, have been reported in the HELPS trial. ${ }^{18}$ It is unclear whether this finding, if real, is an artifact from different coil densities or is caused by coil thrombogenicity or by premature interruption of coiling when operators encounter difficulties or expect hydrogel coil expansion. We did not find a significant difference. In HELPS, differences in occlusion grades were reversed (favoring hydrogel) at the time of follow-up imaging. ${ }^{13}$ Thus, if one keeps in mind that angiographic outcomes judged locally are typically more optimistic than core- laboratory results, ${ }^{26,27}$ these preliminary findings cannot be used to anticipate follow-up imaging results, which remain to be collected, analyzed by the core lab, and reported. While we wait for long-term results from this trial and witness an increasing use of flow diversion for large, wide-neck, and recurrent aneurysms (a practice with as-yet-unknown short- and long-term benefits), ${ }^{28}$ the PRET trial serves as a reminder that difficult aneurysms can be coiled with a safety that will be difficult to improve.

\section{Limitations}

The PRET trial had several limitations. First, operators could not be blinded to coil type. This unavoidable fact may have affected case selection and the use of adjunct devices, coil selection, and perhaps even premature interruption of coiling. Second, different types of hydrogel coils were being manufactured and approved during the course of the trial. This moving-target problem may create difficulties in the interpretation of results. Third, recruitment slowed down during the last 2 years (Fig 1), perhaps because treatment alternatives (such as flow diverters) were increasingly used for the same types of aneurysms. This change possibly introduces a selection bias that could weaken the generalizability of trial results. Fourth, the PRET-2 substudy was interrupted before the target number of patients was enrolled, possibly affecting the power of the study to reach meaningful conclusions for that subgroup at the end of the trial. Fifth, data monitoring was done on-line, with no local site visits to verify the data that were being reported. Finally, postprocedural studies were not imposed by protocol to verify the absence of complications detectable by imaging. These choices made the completion of an important trial at low cost possible. However, the lack of on-site monitoring and of postprocedural brain imaging is not expected to affect the validity of the present conclusions, due to the pragmatic design of the trial, which relies on relatively hard clinical outcomes and the consistency of outcome assessment across several time points. In addition, these perceived deficiencies are expected to affect treatment groups in a balanced manner.

\section{CONCLUSIONS}

There was no significant difference in the safety or immediate efficacy of the procedure between hydrogel and platinum coiling of large and recurrent aneurysms.

\section{PRET Trial Collaborators}

The PRET trial collaborators are listed in the order that participating sites joined the trial, with the number of patients recruited given in parentheses.

CHUM-Notre Dame Hospital, Montreal, Quebec, Canada: Principal Investigators (PIs), Jean Raymond, Alain Weill, and Daniel Roy; Coordinator, Ruby Klink (120). The Methodist Hospital, Houston, Texas: PIs, Richard Klucznik and Orlando Diaz; Coordinator, Marilyn Bautista (12). Kobe City Medical Center General Hospital, Kobe, Japan: PIs, Nobuyuki Sakai and Horotoshi Imamura (4). Medical University of South Carolina, Charleston, South Carolina: PIs, Aquilla Turk and Raymond Turner; Coordinator, Adrian Parker (26). State University of New York at Stony Brook University Medical Center, Stony Brook, New York: PI, Henry Woo; Coordinators, Susan Fiore and Dawn 
Madigan (20). Oregon Health \& Science University, Portland, Oregon: PI, Stanley Barnwell; Coordinator, Sarah Ross-Jamieson (63). Cleveland Clinic, Cleveland, Ohio: PI, Thomas Masaryk; Coordinator, Terese Wheeler (1). Centre Hospitalier Universitaire de Nancy-Hôpital Central, Nancy, France: PI, Serge Bracard; special thanks to Dr Anne Laure Derelle (8). Leeds General Infirmary, Leeds, United Kingdom: PI, Tony Goddard; Coordinator, Jonathan Pearce (also, central coordinator for the UK) (10). The Ottawa Hospital, Ottawa, Ontario, Canada: PI, Marlise Santos; Coordinator, Betty Anne Schwarz (14). Instituto de Neurocirugía Dr Asenjo, Santiago, Chile: PIs, Juan-Gabriel Sordo Jara and Eduardo Bravo (14). University of Florida (Shands Hospital), Gainesville, Florida: PIs, J. Mocco and Brian Hoh; Coordinators, Bree Burks and Nicolle Wilson-Davis (29). University of Virginia Health System, Charlottesville, Virginia: PI, Avery Evans; Coordinators, Claire McKinley and Thomas Tandy (39). Centre Hospitalier Universitaire de Nantes-Hôpital Guillaume et René Laennec, Nantes, France: PI, Hubert Desal (23). West Virginia University Hospital, Morgantown, West Virginia: PI, Jeffrey Carpenter; Coordinator, Jennifer Domico (8). State University of New York Upstate Medical University, Syracuse, New York: PI, Eric Deshaies; Coordinators, Tina Craig, Kim Kasprowicz, Susan Hemingway, and Mark Villwock (10). University of Cincinnati Medical Center, Cincinnati, Ohio: PI, Andrew Ringer; Coordinator, Rebecca Reinert (4). Washington University in St. Louis, St. Louis, Missouri: PI, Christopher Moran; Coordinator, Angela Campbell (6). Queens Medical Centre, Nottingham, United Kingdom: PI, Robert Lenthall; Coordinator, Alison Southam (12). Saint Francis Medical Center, Cape Girardeau, Missouri: PI, Louis Caragine; Coordinators, Adrienne Jones and Kathy O'Howell (2). University of Oklahoma Health Sciences Center, Oklahoma City, Oklahoma: PI, Steven Hoover; Coordinators, Brian Bridges and Bradley Hightower (11). Centre Hospitalier Sainte Anne, Paris, France: PI, Olivier Naggara (2). University of Mississippi Health Care, Jackson, Mississippi: PI, Razvan Buciuc; Coordinator, David Gordy (3). University of Alberta Hospital, Alberta, Canada: PI, Tim E. Darsaut (2). Vanderbilt University Medical Center, Nashville, Tennessee: PI, J. Mocco; Coordinators, Chesney Sarah Oravec and Jessica Sparks Marlin (4).

\section{ACKNOWLEDGMENTS}

First and foremost, we acknowledge the support of patients and their relatives who agreed to participate in the study and who continue to provide us with follow-up data. We also thank the medical, radiologic, and nursing staff of all participating centers. We are grateful to members of the DSMC (Sylvain Lanthier, Allan Fox). Implementing a study such as PRET requires considerable effort over a long time. Special thanks are due to Guylaine Gevry and her team at the PRET Coordination Head Office in Montreal for their outstanding work and indefectible commitment.

Disclosures: Jean Raymond—RELATED: Grant: MicroVention, * Comments: Grant for an investigator-led, unrestricted research funding for the PRET studies (20072012); UNRELATED: Grants/Grants Pending: funding for the Canadian Unruptured Endovascular versus Surgery (CURES) clinical trial from Canadian Institutes of Health research*; Other: participation in a clinical trial,* Comments: an amount per patient for participation in the new generation Hydrogel Endovas- cular Aneurysm Treatment (HEAT) Trial (Northwestern University Feinberg School of Medicine). Ruby Klink-RELATED: Grant: MicroVention funded the PRET trial (industry-funded, investigator-led study). * Stanley L. Barnwell-RELATED: Other: MicroVention, ${ }^{*}$ Comments: support to Stroke Center at OHSU for running trial, not the publication; UNRELATED: Consultancy: MicroVention, Comments: occasional consulting, work unrelated to the trial. Avery J. EvansRELATED: Grant: MicroVention*; UNRELATED: Consultancy: Stryker, MicroVention, Covidien; Grants/Grants Pending: MicroVention, Stryker; Payment for Lectures (including service on Speakers Bureaus): Stryker, MicroVention, Covidien; Patents (planned, pending or issued): patent personally applied for; Payment for Development of Educational Presentations: Stryker; Travel/Accommodations/ Meeting Expenses Unrelated to Activities Listed: Stryker, MicroVention, Covidien. J. Mocco-UNRELATED: Board Membership: Codman Neurovascular (advisory board)*; Consultancy: Lazarus Effect, Medina Medical, Pulsar Vascular, Reverse Medical, Edge Therapeutics; Grants/Grants Pending: National Institutes of Health Funding: NIH 1U01NS086492-01; NIH 1R01NS078828-01A1; Other: Blockade Medical, Medina Medical, Comments: personal investment. Brian L. HohRELATED: Grant: Our institution receives funding for participating in the trial, with payment made by the Centre hospitalier de l'université de Montréal through an unrestricted grant from Microvention, ${ }^{*}$ UNRELATED: Grants/Grants Pending: National Institutes of Health*; Other: Steering Committee for clinical trial for Edge Therapeutics. Aquilla S. Turk—RELATED: Grant: MicroVention, ${ }^{*}$ Comments: study paid per patient for research personnel to collect data for trial purposes; UNRELATED: Consultancy: Penumbra, Stryker, MicroVention, Codman, Medina, Pulsar Vascular; Expert Testimony: defense litigation; Grants/ Grants Pending: Penumbra, ${ }^{\star}$ Stryker, ${ }^{\star}$ MicroVention, ${ }^{*}$ Codman, ${ }^{\star}$ Medina, ${ }^{\star}$ Pulsar Vascular, ${ }^{\star}$ Comments: LARGE trial and POSITIVE trial, enrolling site for multiple other trials; Payment for Lectures (including service on Speakers Bureaus): Penumbra, MicroVention; Stock/Stock Options: Pulsar Vascular, Medina, Lazarus Effect; Travel/Accommodations/Meeting Expenses Unrelated to Activities Listed: Penumbra, ${ }^{*}$ Covidien, ${ }^{\star}$ Codman, ${ }^{*}$ Stryker. Raymond D. Turner-RELATED: Grant: Codman,* Covidien,* MicroVention,* Stryker,* Blockade Medical,* Reverse Medical*; Consulting Fee or Honorarium: MicroVention, Codman, Covidien, Blockade Medical, Pulsar Vascular, Reverse Medical. David FiorellaRELATED: Grant: MicroVention/Terumo*; UNRELATED: Consultancy: Covidien/ ev3, Codman/JNJ; Grants/Grants Pending: Siemens Medical Imaging, ${ }^{*}$ Sequent Medical,* Comments: LVIS IDE study PI, Sequent WEB IDE trial PI; Patents (planned, pending or issued): Codman/JNJ; Royalties: Codman/JNJ; Travel/Accommodations/Meeting Expenses Unrelated to Activities Listed: Penumbra. Serge Bracard-UNRELATED: Grants/Grants Pending: French Ministry of Health Program STIC,* Comments: randomized study THRACE on thrombectomy in stroke. Alain Weill-UNRELATED: Grants/Grants Pending: fellowship grants from Codman, * Stryker, * Covidien*; Payment for Lectures (including service on Speakers Bureaus): MicroVention, ${ }^{*}$ for lecture given at AAFITN 2014 on bailout strategies in aneurysm coiling. Daniel Roy-RELATED: Grant: MicroVention,* Comments: grant support for the study. No role in design, data management, or manuscript. *Money paid to the institution.

\section{REFERENCES}

1. Molyneux A, Kerr R, Stratton I, et al. International Subarachnoid Aneurysm Trial (ISAT) of neurosurgical clipping versus endovascular coiling in 2143 patients with ruptured intracranial aneurysms: a randomised trial. Lancet 2002;360:1267-74

2. Molyneux AJ, Kerr RS, Yu LM, et al. International Subarachnoid Aneurysm Trial (ISAT) of neurosurgical clipping versus endovascular coiling in 2143 patients with ruptured intracranial aneurysms: a randomised comparison of effects on survival, dependency, seizures, rebleeding, subgroups, and aneurysm occlusion. Lancet 2005;366:809-17

3. Ferns SP, Sprengers ME, van Rooij WJ, et al. Coiling of intracranial aneurysms: a systematic review on initial occlusion and reopening and retreatment rates. Stroke 2009;40:e523-29

4. Carat Investigators. Rates of delayed rebleeding from intracranial aneurysms are low after surgical and endovascular treatment. Stroke 2006;37:1437-42

5. Campi A, Ramzi N, Molyneux AJ, et al. Retreatment of ruptured cerebral aneurysms in patients randomized by coiling or clipping in the International Subarachnoid Aneurysm Trial (ISAT). Stroke 2007;38:1538-44

6. Raymond J. Counterpoint: has the last word been said? AJNR Am J Neuroradiol 2009;30:1649-52

7. Darsaut TE, Findlay JM, Raymond J. The design of the Canadian 
UnRuptured Endovascular versus Surgery (CURES) trial. Can J Neurol Sci 2011;38:236-41

8. Raymond J, Molyneux AJ, Fox AJ, et al. The TEAM trial: safety and efficacy of endovascular treatment of unruptured intracranial aneurysms in the prevention of aneurysmal hemorrhages-a randomized comparison with indefinite deferral of treatment in 2002 patients followed for 10 years. Trials 2008;9:43

9. Naggara ON, Lecler A, Oppenheim C, et al. Endovascular treatment of intracranial unruptured aneurysms: a systematic review of the literature on safety with emphasis on subgroup analyses. Radiology 2012;263:828-35

10. White PM, Raymond J. Endovascular coiling of cerebral aneurysms using "bioactive" or coated-coil technologies: a systematic review of the literature. AJNR Am J Neuroradiol 2009;30:219-26

11. McDougall CG, Johnston SC, Gholkar A, et al. Bioactive versus bare platinum coils in the treatment of intracranial aneurysms: the MAPS (Matrix and Platinum Science) Trial. AJNR Am J Neuroradiol 2014;35:935-42

12. Molyneux AJ, Clarke A, Sneade M, et al. Cerecyte Coil Trial: angiographic outcomes of a prospective randomized trial comparing endovascular coiling of cerebral aneurysms with either Cerecyte or bare platinum coils. Stroke 2012;43:2544-50

13. White PM, Lewis SC, Gholkar A, et al. Hydrogel-coated coils versus bare platinum coils for the endovascular treatment of intracranial aneurysms (HELPS): a randomised controlled trial. Lancet 2011;377:1655-62

14. Raymond J, Roy D, White PM, et al. A randomized trial comparing platinum and hydrogel-coated coils in Patients Prone to Recurrence after Endovascular Treatment (the PRET Trial). Interv Neuroradiol 2008; $14: 73-83$

15. Raymond J, Guilbert F, Weill A, et al. Long-term angiographic recurrences after selective endovascular treatment of aneurysms with detachable coils. Stroke 2003;34:1398-403

16. Roy D, Milot G, Raymond J. Endovascular treatment of unruptured aneurysms. Stroke 2001;32:1998-2004

17. Coley S, Sneade M, Clarke A, et al. Cerecyte coil trial: procedural safety and clinical outcomes in patients with ruptured and unruptured intracranial aneurysms. AJNR Am J Neuroradiol 2012;33:474-80
18. White PM, Lewis SC, Nahser H, et al. HydroCoil Endovascular Aneurysm Occlusion and Packing Study (HELPS trial): procedural safety and operator-assessed efficacy results. AJNR Am J Neuroradiol 2008;29:217-23

19. Cloft HJ, Kallmes DF. Cerebral aneurysm perforations complicating therapy with Guglielmi detachable coils: a meta-analysis. AJNR Am J Neuroradiol 2002;23:1706-09

20. Nguyen TN, Raymond J, Guilbert F, et al. Association of endovascular therapy of very small ruptured aneurysms with higher rates of procedure-related rupture. J Neurosurg 2008;108:1088-92

21. Pelz DM, Lownie SP, Fox AJ. Thromboembolic events associated with the treatment of cerebral aneurysms with Guglielmi detachable coils. AJNR Am J Neuroradiol 1998;19:1541-47

22. Meyers PM, Lavine SD, Fitzsimmons BF, et al. Chemical meningitis after cerebral aneurysm treatment using two second-generation aneurysm coils: report of two cases. Neurosurgery 2004;55:1222

23. Turner RD, Byrne JV, Kelly ME, et al. Delayed visual deficits and monocular blindness after endovascular treatment of large and giant paraophthalmic aneurysms. Neurosurgery 2008;63:469-74, discussion $474-75$

24. Xu DS, Hurley MC, Batjer HH, et al. Delayed cranial nerve palsy after coiling of carotid cavernous sinus aneurysms: case report. Neurosurgery 2010;66:E1215-16

25. Im SH, Han MH, Kwon BJ, et al. Aseptic meningitis after embolization of cerebral aneurysms using hydrogel-coated coils: report of three cases. AJNR Am J Neuroradiol 2007;28:511-12

26. Tollard E, Darsaut TE, Bing F, et al. Outcomes of endovascular treatments of aneurysms: observer variability and implications for interpreting case series and planning randomized trials. AJNR Am J Neuroradiol 2012;33:626-31

27. Rezek I, Lingineni RK, Sneade M, et al. Differences in the angiographic evaluation of coiled cerebral aneurysms between a core laboratory reader and operators: results of the Cerecyte Coil Trial. AJNR Am J Neuroradiol 2014;35:124-27

28. Becske T, Kallmes DF, Saatci I, et al. Pipeline for uncoilable or failed aneurysms: results from a multicenter clinical trial. Radiology 2013;267: $858-68$ 